Low-energy charge transfer excitations in $\mathrm{NiO}$

This content has been downloaded from IOPscience. Please scroll down to see the full text. 2012 IOP Conf. Ser.: Mater. Sci. Eng. 38012007

(http://iopscience.iop.org/1757-899X/38/1/012007)

View the table of contents for this issue, or go to the journal homepage for more

Download details:

IP Address: 212.193.78.1

This content was downloaded on 27/03/2015 at 08:47

Please note that terms and conditions apply. 


\title{
Low-energy charge transfer excitations in $\mathrm{NiO}$
}

\author{
V I Sokolov ${ }^{1}$, V A Pustovarov ${ }^{2}$, V N Churmanov ${ }^{2}$, V Yu Ivanov ${ }^{2}$, A \\ Ye Yermakov ${ }^{1}$, M A Uimin ${ }^{1}$, N B Gruzdev ${ }^{1}$, P S Sokolov ${ }^{3}$, A N \\ Baranov $^{3}$ and A S Moskvin ${ }^{4}$ \\ ${ }^{1}$ Institute of Metal Physics UD RAS, S. Kovalevskaya Str. 18, 620990, Ekaterinburg, Russia \\ ${ }^{2}$ Ural Federal University, Mira Str., 19, 620002 Ekaterinburg, Russia \\ ${ }^{3}$ Lomonosov Moscow State University, 119991, Moscow, Russia \\ ${ }^{4}$ Department of Theoretical Physics, Institute of Natural Science, Ural Federal University, \\ Lenin Str., 51, 620083 Ekaterinburg, Russia \\ E-mail: alexandr.moskvin@usu.ru
}

\begin{abstract}
Comparative analysis of photoluminescence (PL) and photoluminescence excitation (PLE) spectra of $\mathrm{NiO}$ poly- and nanocrystals in the spectral range $2-5.5 \mathrm{eV}$ reveals two PLE bands peaked near 3.7 and $4.6 \mathrm{eV}$ with a dramatic rise in the low-temperature PLE spectral weight of the $3.7 \mathrm{eV}$ PLE band in the nanocrystalline $\mathrm{NiO}$ as compared with its polycrystalline counterpart. In frames of a cluster model approach we assign the $3.7 \mathrm{eV}$ PLE band to the low-energy bulk-forbidden $p$ - $d\left(t_{1 g}(\pi)-e_{g}\right)$ charge transfer $(\mathrm{CT})$ transition which becomes the allowed one in the nanocrystalline state while the $4.6 \mathrm{eV}$ PLE band is related to a bulk allowed $d-d\left(e_{g}-e_{g}\right)$ CT transition scarcely susceptible to the nanocrystallization. The PLE spectroscopy of the nanocrystalline materials appears to be a novel informative technique for inspection of different $\mathrm{CT}$ transitions.
\end{abstract}

\section{Introduction}

Despite several decades of studies there is still no literature consensus on the detailed electronic structure of the prototype $3 \mathrm{~d}$ oxide $\mathrm{NiO}$, in particular, the character of the low-energy charge transfer excitations. $\mathrm{NiO}$ has long been viewed as a prototype "Mott insulator" [1] with a gap formed by intersite $d$ - $d$ charge transfer (CT) transitions, however, this view was later replaced by that of a "CT insulator" [2] with gap formed by $p$ - $d$ CT transitions. At present we have no comprehensive assignment of intensive spectral features in $\mathrm{NiO}$ to different $p$ - $d$ or $d-d$ CT transitions. The main reason for this is that the absorption coefficient rises steeply above $3.5 \mathrm{eV}$ and reaches a giant value of $10^{6} \mathrm{~cm}^{-1}$ at and above $4 \mathrm{eV}[3]$ that complicates the detailed absorption and reflection measurements particularly near the fundamental edge. This difficulty can be avoided in part by making use of specific techniques such as electroreflectance measurements [4] or nonlinear absorption measurements [5] that are particularly effective for location of the forbidden transitions.

Main goal of our paper is to show with $\mathrm{NiO}$ as an example that the photoluminescence excitation (PLE) spectroscopy of the nanocrystalline materials appears to be a novel informative technique for inspection of different charge transfer transitions. Indeed, on the one hand, nanocrystalline state gives rise to a noticeable modification of the optical response of materials, in particular, due to an enhanced role of the surface induced local structural distortions resulting in a shift and splitting of the energy levels, and, mainly, in a liberalization of the 
selection rules. It means that optical response of nanocrystalline materials can reveal electronhole excitations unresolved in bulk spectra. On the other hand, the photoluminescence (PL) spectroscopy can give a profound insight into the electronic structure, electron-hole excitations and their relaxation in the lattice. As regards nanocrystalline $\mathrm{NiO}$, its optical properties both in absorption and emission have been scarcely investigated. To the best of our knowledge the photoluminescence studies of nanocrystalline $\mathrm{NiO}$ are restricted by just two papers $[6,7]$. Volkov et al. [6] have performed the steady-state absorption and time-resolved luminescence measurements for nanoclustered $\mathrm{NiO}$ samples with the particle size of $0.6 \mathrm{~nm}$. The authors of Ref. [7] have presented certain controversial results of absorption and photoluminescence measurements for $\mathrm{NiO}$ nanocrystals with the particle size of $40 \mathrm{~nm}$. However, in the both researches there is no inspection of the PL photoexcitation over the CT band, though such a study together with the comparative analysis of the poly- and nanocrystalline NiO PL and PLE spectra can be an instructive tool to elucidate the mechanism of the CT transitions and the spectral selectivity of the photoluminescence. These issues did motivate our studies of the PL and PLE spectra in nanocrystalline $\mathrm{NiO}$ samples as compared with polycrystalline samples.

\section{Experimental results}

In order to make clear the effect of nanocrystallization the PL measurements were made both on conventional polycrystalline and nanocrystalline samples of $\mathrm{NiO}$. As a starting material for polycrystalline $\mathrm{NiO}$ we have used the commercially available powder of $\mathrm{NiO}$ (99\%; Prolabo) which has been pressed into pellets under pressure of about $0.125 \mathrm{GPa}$ and placed into gold capsules. Quenching experiments at $7.7 \mathrm{GPa}$ and $1000-1100 \mathrm{~K}$ have been performed using a toroid-type high-pressure apparatus. Detailes of experimental technique and calibration are described elsewhere [8]. Electron microscopy analysis shows the samples to be dense poreless oxide ceramics with rock salt cubic structure and grain size of about 10-20 $\mu \mathrm{m}$. Nanocrystalline samples of $\mathrm{NiO}$ with a particle size of $25 \mathrm{~nm}$ were produced by the gas-condensation synthesis method [9] and annealed in air at a temperature of $350^{\circ} \mathrm{C}$ for an 24 hour. The samples for our measurements were produced by tablet pressing at a pressure of $0.5 \mathrm{GPa}$. The PL and PLE spectra were measured in the range (2-5.5) eV using two double DMP-4 prism monochromators (a reciprocal linear dispersion of $10 \AA / \mathrm{mm}$ near $5 \mathrm{eV}$ ), an R6358-10 (Hamamatsu) photomultiplier tube, and a photon-counting system [10,11]. A deuterium DDS-400 lamp was used for excitation. The PLE spectra were normalized to the same number of photons incident on the sample using yellow phosphor, which has an energy-independent PL quantum yield in the energy range under investigation. The PL spectra are presented without any normalization to the spectral sensitivity of the optical section. Typical PL spectra of poly- and nanocrystalline $\mathrm{NiO}$ samples measured at room temperature and low temperature $\mathrm{T}=90 \mathrm{~K}$ under excitation energies suggestive the CT band excitation $\left(E_{e x c}>E_{g}\right)$ are presented in upper and lower panels of Fig. 1, respectively. Such a broad luminescence band in the region $2-3.5 \mathrm{eV}$ was observed in earlier PL measurements performed with UV excitation below and near optical gap [12, 6, 13]. The lowenergy excitation $\left(E_{e x c} \approx 2.4 \mathrm{eV}\right)$ below the charge-transfer gap stimulates a photoemission in single-crystal $\mathrm{NiO}$ with two maxima, one at $1.5-1.6 \mathrm{eV}$ and a larger one at $2.2-2.3 \mathrm{eV}$ [14]. Green luminescence band with a maximum around $2.25 \mathrm{eV}$ has been observed in nanoclustered $\mathrm{NiO}$ at $E_{\text {exc }} \leq 2.95 \mathrm{eV}[6]$. The observed emission bands in the visible and near infrared spectral ranges are usually attributed to $\mathrm{Ni}^{2+}$ intrasite, or crystal field $d$ - $d$ transitions. In particular, the main green luminescence band peaked near $2.3 \mathrm{eV}$ is attributed to a Stokesshifted ${ }^{1} T_{2 g}(D) \rightarrow{ }^{3} A_{2 g}(F)$ transition while the low-energy band peaked near $1.5 \mathrm{eV}$ is related to ${ }^{1} E_{g}(D) \rightarrow{ }^{3} A_{2 g}(F)$ transition [14] (see Ref. [3] for the spectrum of the crystal field $d$ - $d$ transitions). However, the photoluminescence spectra of bulk single crystals and ceramics of $\mathrm{NiO}$ under $3.81 \mathrm{eV}$ photoexcitation [13] suggestive the band gap excitation have revealed in addition to the green band a more intensive broad violet PL band with a maximum around 

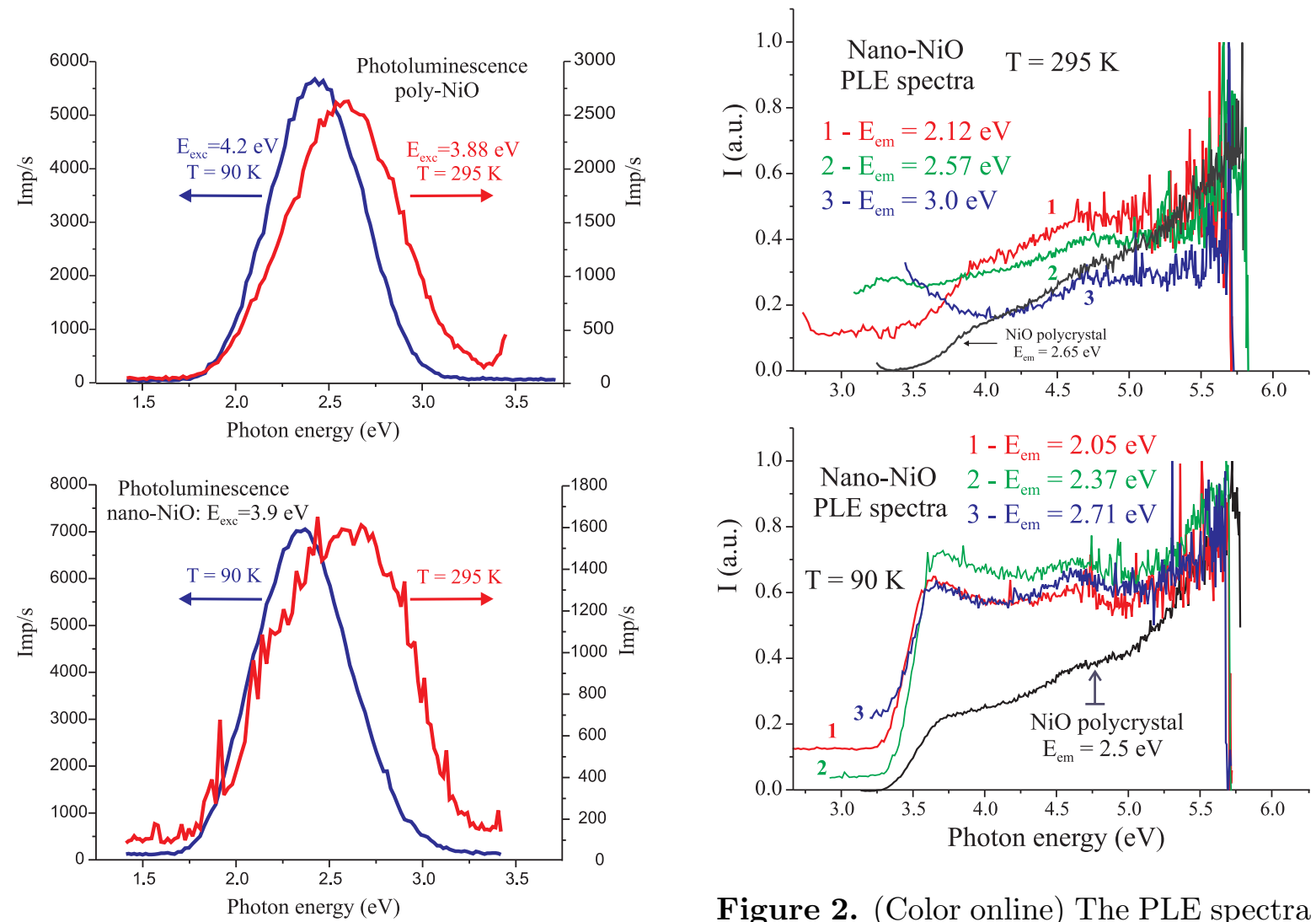

Figure 1. (Color online) Photoluminescence spectra of polycrystalline (upper panel) and nanocrystalline (lower panel) $\mathrm{NiO}$ samples at two temperatures and $E_{\text {exc }}>E_{g}$.

Figure 2. (Color online) The PLE spectra of nanocrystalline $\mathrm{NiO}$ at two temperatures $(\mathrm{T}=295 \mathrm{~K}$, upper panel, $\mathrm{T}=90 \mathrm{~K}$, lower panel) and different emission energies. For comparison in the both panels shown are the PLE spectra of polycrystalline $\mathrm{NiO}$ at corresponding temperatures.

$3 \mathrm{eV}$. The band was related to a $p$ - $d$ charge transfer. Radiative recombination of carriers in powdered pellets of $\mathrm{NiO}$ under UV excitation with $E_{\text {exc }}=4.43 \mathrm{eV}(280 \mathrm{~nm})$ higher than the CT gap consists at $10 \mathrm{~K}$ of a broad intensive band peaked at $2.8 \mathrm{eV}$ with a shoulder centered at about $3.2 \mathrm{eV}$ [12]. At variance with Ref. [13] the PL emission bands observed in the work [12] were attributed to the crystal field $d$ - $d$ transitions. Fig. 1 clearly shows the blue shift of the PL band with increasing the temperature both for polycrystalline and nanocrystalline samples of $\mathrm{NiO}$. Such a behavior was noticed in earlier measurements for polycrystalline samples of $\mathrm{NiO}[12]$. Furthermore, the detailed temperature measurements [12] indicate that the broad PL spectra of $\mathrm{NiO}$ consist of several components with relative intensity dependent on the temperature and excitation energy.

Different kinetic properties, seemingly different temperature behavior [12] point to different relaxation channels governing the green and violet luminescence. Despite the PL spectra for nanocrystalline and polycrystalline $\mathrm{NiO}$ have similar lineshape, it is worth noting a clear red shift of the low-temperature PL spectra and a visible assymetry of the high-temperature PL spectra for nano-NiO.

Main results of our paper are related with the PLE spectral measurements in range 2$5.5 \mathrm{eV}$ presented in Fig. 2. The polycrystalline $\mathrm{NiO}$ sample reveals a rather structureless $\mathrm{PL}$ excitation spectrum with a steep spectral weight rise from 3.3 towards $6 \mathrm{eV}$ and scarcely marked 
weak features near 3.7 and $4.6 \mathrm{eV}$. The low-energy feature becomes more pronounced at low temperatures $\mathrm{T}=90 \mathrm{~K}$. The room temperature PLE spectra for nanocrystalline $\mathrm{NiO}$ corroborates the presence of at least the $4.6 \mathrm{eV}$ feature while the obscure lower energy spectral behavior strongly depends on the emission energy. Just another lower energy spectral behavior in PLE spectra for nanocrystalline $\mathrm{NiO}$ is observed at low temperature $\mathrm{T}=90 \mathrm{~K}$ (see lower panel in Fig. 2). Here we see a dramatic rise in the PLE spectral weight with a peak at excitation energy $E_{\text {exc }} \approx 3.7 \mathrm{eV}$ that points to the energy $E_{g}^{P L E} \approx 3.5 \mathrm{eV}$ as a specific PLE gap for the both polyand nanocrystalline $\mathrm{NiO}$. Interestingly that the spectral weight and position of the $4.6 \mathrm{eV}$ PLE feature seem to weakly alter in between poly- and nanocrystalline $\mathrm{NiO}$ samples. Thus, we are drawn to the conclusion that the $3.7 \mathrm{eV}$ band can be most likely related with the bulk-forbidden $\mathrm{CT}$ transition which becomes the allowed one in the nanocrystalline state while the $4.6 \mathrm{eV}$ PLE band is believed to be related with a bulk allowed CT transition scarcely susceptible to the nanocrystallization. Such an attribution is supported by several arguments:

1. Electroreflectance spectra for bulk $\mathrm{NiO}[4]$ which detect the dipole-forbidden $\mathrm{CT}$ transitions clearly point to a forbidden transition peaked near $3.7 \mathrm{eV}$ missed in reflectance and absorption spectra $[3,15,16]$.

2. At variance with the bulk $\mathrm{NiO}$ a clearly visible intensive peak near $3.6-3.7 \mathrm{eV}$ has been observed in absorption spectra of $\mathrm{NiO}$ nanoparticles $[6,7,17]$. Its intensity points to the $\mathrm{CT}$ origin.

3. Peak near $3.8 \mathrm{eV}$ has been observed in nonlinear absorption spectra of $\mathrm{NiO}[5]$.

4. Photoconductivity threshold in bulk $\mathrm{NiO}$ has been observed at $3.7 \mathrm{eV}$ [18].

5. A rather strong $\mathrm{CT}$ band peaked at $4.5 \mathrm{eV}$ is clearly visible in the absorption of thin $\mathrm{NiO}$ films [19], RIXS spectra [20, 21], the reflectance spectra (4.3 eV) [15], the nonlinear absorption spectra [5]. All this points to dipole-allowed CT transition.

Below we will address the microscopic origin of the CT transitions defining the PLE bands at 3.7 and $4.6 \mathrm{eV}$.

\section{Discussion}

Despite the nature of radiative and nonradiative transitions in strongly correlated $3 d$ oxides is far from full understanding some reliable semiquantitative predictions can be made in frames of a simple cluster approach (see, e.g. Refs. [22] and references therein). The method provides a clear physical picture of the complex electronic structure and the energy spectrum, as well as the possibility of a quantitative modelling. In a certain sense the cluster calculations might provide a better description of the overall electronic structure of insulating $3 \mathrm{~d}$ oxides than the band structure calculations, mainly due to a better account for correlation effects. Starting with octahedral $\mathrm{NiO}_{6}$ complex with the point symmetry group $O_{h}$ we deal with five $\mathrm{Ni} 3 \mathrm{~d}$ and eighteen oxygen $\mathrm{O} 2 \mathrm{p}$ atomic orbitals forming both hybrid $\mathrm{Ni} 3 \mathrm{~d}-\mathrm{O} 2 \mathrm{p}$ bonding and antibonding $e_{g}$ and $t_{2 g}$ molecular orbitals (MO), and purely oxygen nonbonding $a_{1 g}(\sigma), t_{1 g}(\pi), t_{1 u}(\sigma), t_{1 u}(\pi)$, $t_{2 u}(\pi)$ orbitals. Typically for the octahedral $\mathrm{MeO}_{6}$ clusters the non-bonding $t_{1 g}(\pi)$ oxygen orbital has the highest energy and forms the first electron removal oxygen state while other nonbonding oxygen $\pi$-orbitals, $t_{2 u}(\pi), t_{1 u}(\pi)$, and $\sigma$-orbital $t_{1 u}(\sigma)$ have lower energy with the energy separation $\sim 1 \mathrm{eV}$ in between $[22]$.

The $p$ - $d$ CT transitions in $\mathrm{NiO}_{6}^{10-}$ center are related with the transfer of the $\mathrm{O} 2 \mathrm{p}$ electron to the partially filled $3 \mathrm{~d} e_{g}$-subshell with formation on the Ni-site of the $\left(t_{2 g}^{6} e_{g}^{3}\right)$ configuration of nominal $\mathrm{Ni}^{+}$ion isoelectronic to the well-known Jahn-Teller $\mathrm{Cu}^{2+}$ ion. Yet actually instead of a single $p$ - $d$ CT transition we arrive at a series of $\mathrm{O} 2 \mathrm{p} \gamma \rightarrow \mathrm{Ni} 3 \mathrm{~d} e_{g} \mathrm{CT}$ transitions forming a complex $p$ - $d$ CT band. It should be noted that each single electron transition gives rise to two many-electron transitions. The band starts with the dipole-forbidden $t_{1 g}(\pi) \rightarrow e_{g}$, or ${ }^{3} A_{2 g} \rightarrow{ }^{3} T_{1 g},{ }^{3} T_{2 g}$ transitions, then includes two formally dipole-allowed so-called $\pi \rightarrow \sigma p$ - $d \mathrm{CT}$ transitions, weak $t_{2 u}(\pi) \rightarrow e_{g}$, and relatively strong $t_{1 u}(\pi) \rightarrow e_{g}$ CT transitions, respectively, 
each giving rise to ${ }^{3} A_{2 q} \rightarrow{ }^{3} T_{1 u},{ }^{3} T_{2 u}$ transitions. Finally main $p$ - $d$ CT band is ended by the strongest dipole-allowed $\sigma \rightarrow \sigma t_{1 u}(\sigma) \rightarrow e_{g}\left({ }^{3} A_{2 g} \rightarrow{ }^{3} T_{1 u},{ }^{3} T_{2 u}\right)$ CT transition. Above estimates predict the separation between partial $p$ - $d$ bands to be $\sim 1 \mathrm{eV}$. Thus, if the most intensive CT band with a maximum around $7 \mathrm{eV}$ observed in RIXS spectra $[20,21]$ to attribute to the strongest dipole-allowed $\mathrm{O} 2 \mathrm{p} t_{1 u}(\sigma) \rightarrow \mathrm{Ni} 3 \mathrm{~d} e_{g} \mathrm{CT}$ transition then one should expect the low-energy $p$ - $d$ CT counterparts with maximuma around 4,5 , and $6 \mathrm{eV}$ respectively, which are related to dipoleforbidden $t_{1 g}(\pi) \rightarrow e_{g}$, weak dipole-allowed $t_{2 u}(\pi) \rightarrow e_{g}$, and relatively strong dipole-allowed $t_{1 u}(\pi) \rightarrow e_{g} \mathrm{CT}$ transitions, respectively. It is worth noting that the $\pi \rightarrow \sigma p$ - $d \mathrm{CT} t_{1 u}(\pi)-e_{g}$ transition borrows a portion of intensity from the strongest dipole-allowed $\sigma \rightarrow \sigma t_{1 u}(\sigma)-e_{g}$ CT transition because the $t_{1 u}(\pi)$ and $t_{1 u}(\sigma)$ states of the same symmetry are partly hybridized due to p-p covalency and overlap. Interestingly that this assignement finds a strong support in the reflectance $(4.9,6.1$, and $7.2 \mathrm{eV}$ for allowed $p$ - $d$ CT transitions) [15] and, particularly, in electroreflectance spectra $[15,4]$ which detect dipole-forbidden transitions. Indeed, the spectra clearly point to a forbidden transition peaked near $3.7 \mathrm{eV}$ (missed in reflectance spectra) which thus defines a $p$ - $d$ character of the optical CT gap and can be related with the on-set transition for the whole complex $p$ - $d$ CT band. It should be noted that a peak near $3.8 \mathrm{eV}$ has been observed in nonlinear absorption spectra of $\mathrm{NiO}$ [5]. A rather strong $p(\pi)-d$ CT band peaked at $6.3 \mathrm{eV}$ is clearly visible in the absorption spectra of $\mathrm{MgO}: \mathrm{Ni}[16]$.

As a result of the $p$ - $d$ CT transition, a photo-generated electron localizes on a $\mathrm{Ni}^{2+}$ ion forming Jahn-Teller $3 \mathrm{~d}^{9}$, or $\mathrm{Ni}^{1+}$ configuration, while a photo-generated hole can move more or less itinerantly in the $\mathrm{O} 2 \mathrm{p}$ valence band determining the hole-like photoconductivity [18]. The most effective channel of the recombinational relaxation for the spin-triplet $p$ - $d$ CT states $t_{2 g}^{6} e_{g}^{3} \gamma(\pi)\left(\gamma(\pi)=t_{1 g}(\pi), t_{2 u}(\pi), t_{1 u}(\pi)\right)$ implies the $\pi \rightarrow \pi$ transfer $t_{2 g} \rightarrow \gamma(\pi)$ with formation of excited spin-triplet ${ }^{3} T_{1 g}$ or ${ }^{3} T_{2 g}$ states of the $t_{2 g}^{5} e_{g}^{3}$ configuration of $\mathrm{Ni}^{2+}$ ion followed by final relaxation to lowest singlet terms ${ }^{1} T_{2 g}$ and ${ }^{1} E_{g}$ producing green and red luminescence, respectively. Obviously, this relaxation is strongly enhanced by any symmetry breaking effects lifting or weakening the selection rules. It means the three $\pi \rightarrow \sigma p$ - $d$ CT transitions $t_{1 g}(\pi) \rightarrow e_{g}$, $t_{2 u}(\pi) \rightarrow e_{g}$, and $t_{1 u}(\pi) \rightarrow e_{g}$ are expected to effectively stimulate the green luminescence due to large effective mass of $\pi$-oxygen holes and a well-localized and long-lived character of the $p$ - $d$ excitation. On the other hand, the most intensive $\sigma \rightarrow \sigma p$ - $d$ CT transition $t_{1 g}(\sigma) \rightarrow e_{g}$ appears to be significantly less effective due to small effective mass of the $\sigma$-oxygen holes and relatively short lifetime of the respective unstable $p$ - $d$ CT state.

Along with the $p$ - $d \mathrm{CT}$ transitions an important contribution to the optical response of strongly correlated $3 \mathrm{~d}$ oxides can be related with strong $d$ - $d$ CT, or Mott transitions [22]. In $\mathrm{NiO}$ one expects a strong $d$ - $d \mathrm{CT}$ transition related with the $\sigma-\sigma$-type $e_{g}-e_{g}$ charge transfer $t_{2 g}^{6} e_{g}^{2}+t_{2 g}^{6} e_{g}^{2} \rightarrow t_{2 g}^{6} e_{g}^{3}+t_{2 g}^{6} e_{g}^{1}$ between $n n n \mathrm{Ni}$ sites with the creation of electron $\left[\mathrm{NiO}_{6}\right]^{11-}$ and hole $\left[\mathrm{NiO}_{6}\right]^{9-}$ centers (electron-hole dimer), or nominally $\mathrm{Ni}^{+}$and $\mathrm{Ni}^{3+}$ ions. This unique anti-Jahn-Teller transition ${ }^{3} A_{2 g}+{ }^{3} A_{2 g} \rightarrow{ }^{2} E_{g}+{ }^{2} E_{g}$ creates a $d$ - $d$ CT exciton self-trapped in the lattice due to electron-hole attraction and strong "double" Jahn-Teller effect for the both electron and hole centers.

Strong dipole-allowed Franck-Condon $d\left(e_{g}\right)-d\left(e_{g}\right) \mathrm{CT}$ transition in $\mathrm{NiO}$ manifests itself as a strong spectral feature near $4.5 \mathrm{eV}$ clearly visible in the absorption of thin $\mathrm{NiO}$ films [19], the RIXS spectra [20, 21], the reflectance spectra $(4.3 \mathrm{eV})[15]$, the nonlinear absorption spectra (4.1-4.3-4.5eV) [5]. Such a strong absorption near $4 \mathrm{eV}$ is beyond the predictions of the $p$ - $d$ $\mathrm{CT}$ model and indeed is lacking in absorption spectra of $\mathrm{MgO}: \mathrm{Ni}[16]$. Accordingly, the CT gap in $\mathrm{NiO}$ is believed to be formed by a superposition of the electro-dipole forbidden $p$ - $d$ $\left(t_{1 g}(\pi) \rightarrow e_{g}\right)$ and allowed $d\left(e_{g}\right)-d\left(e_{g}\right) \mathrm{CT}$ transitions with close energies that is $\mathrm{NiO}$ is close to the intermediate regime of the Zaanen-Sawatzky-Allen scheme. Thus we see that simple cluster model unambiguously points to the low-energy bulk-forbidden $p$ - $d\left(t_{1 g}(\pi) \rightarrow e_{g}\right)$ and allowed $d\left(e_{g}\right)-d\left(e_{g}\right) \mathrm{CT}$ transitions to be transitions responsible for the 3.7 and $4.6 \mathrm{eV}$ bands revealed 
by the PLE spectroscopy of nanocrystalline NiO.

\section{Conclusion}

In summary, we have measured the photoluminescence and photoluminescence excitation spectra of $\mathrm{NiO}$ poly- and nanocrystals in the spectral range $2-5.5 \mathrm{eV}$. The PL spectra for both types of samples have a very similar spectral shape with broad emission bands centered at about $2.5 \mathrm{eV}$. The PLE spectra reveal two PLE bands peaked near 3.7 and $4.6 \mathrm{eV}$ with a dramatic rise in the low-temperature PLE spectral weight of the $3.7 \mathrm{eV}$ PLE band in the nanocrystalline $\mathrm{NiO}$ as compared with its polycrystalline counterpart. We have performed a cluster model analysis of the CT transitions and assigned the $3.7 \mathrm{eV}$ PLE band to the low-energy bulk-forbidden $p$ - $d$ $\left(t_{1 g}(\pi)-e_{g}\right) \mathrm{CT}$ transition which becomes the allowed one in the nanocrystalline state while the 4.6 eV PLE band is related to the bulk allowed $d-d\left(e_{g}-e_{g}\right)$ CT transition scarcely susceptible to the nanocrystallization. For the first time we succeded in distinct separating the contributions of the low-energy $p(\pi)-d$ and $d$ - $d$ CT transitions to the fundamental absorption of $\mathrm{NiO}$ and show that $\mathrm{NiO}$ is close to the intermediate regime of the Zaanen-Sawatzky-Allen scheme. Thus, the PLE spectroscopy of the nanocrystalline materials is shown to be a novel informative technique for inspection of different CT transitions.

\section{Acknowledgments}

The authors are grateful to R.V. Pisarev for stimulating discussions. This work was partially supported by the Ural Branch RAS (Grant No. 12-U-2-1030) and RFBR (Grants No. 10-02-96032 and 12-02-01039).

\section{References}

[1] Brandow B 1977 Adv. Phys. 26 651; 2008 J. Phys.: Conf. Ser. 108012009

[2] Zaanen J, Sawatzky G A and Allen J W 1985 Phys. Rev. Lett. 55418

[3] Newman R and Chrenko R M 1959 Phys. Rev. 1141507

[4] McNatt J L 1969 Phys. Rev. Lett. 23 915; Glosser R. and Walker W C 1971 Solid State Commun. 91599

[5] Shablaev S I and Pisarev R V 2003 Physics of the Solid State 451742

[6] Volkov V V, Wang Z L and Zou B S 2001 Chemical Phys. Lett. 337117

[7] Chakrabarty S and Chatterjee K 2009 Journal of Physical Sciences 13245

[8] Baranov A N, Sokolov P S, Kurakevich O O, Tafeenko V A, Trots D. and Solozhenko V L 2008 High Pressure Research 28515

[9] Sokolov V I, Yermakov A E, Uimin M A, Mysik A A, Vykhodets V B, Kurennykh T E, Gaviko V S, Shchegoleva N N and Gruzdev N B 2007 JETP 10565

[10] Sokolov V I, Yermakov A E, Uimin M A, Mysik A A, Pustovarov V A, Chukichev M V and Gruzdev N B 2009 J. Lumin. 1291771

[11] Gruzdev N B, Sokolov V I, Yermakov A E, Uimin M A, Mysik A A and Pustovarov V A 2010 JETP 111 231

[12] Díaz-Guerra C, Remón A, García J A and Piqueras J 1997 Phys. Stat. Solidi (a) 163497

[13] Mochizuki S and Saito T 2009 Physica B 4044850

[14] Kuzmin A, Mironova-Ulmane N and Rochin S 2003 Proceedings SPIE 512261

[15] Powell R J and Spicer W E 1970 Phys. Rev. B 22182

[16] Blazey K W 1977 Physica B 8947

[17] Absorbance spectrum in Ref. [7] distinctly points to an absorption peak near $3.7 \mathrm{eV}$, however, with overall view of the spectrum to be at odds with literature data

[18] Ksendzov Ja M and Drabkin I A 1965 Sov. Phys. Solid State 71519

[19] Rossi C E and Paul W 1969 J. Phys. Chem. Solids 302295

[20] Duda L-C, Schmitt T, Magnuson M, Forsberg J, Olsson A, Nordgren J, Okada K and Kotani A 2006 Phys. Rev. Lett. 96067402

[21] Hiraoka N, Okamura H, Ishii H, Jarrige I, Tsuei K D and Cai Y Q 2009 Eur. Phys. J. B 70157

[22] Moskvin A S 2002 Phys. Rev. B 65 205113; Optics and Spectroscopy 111 403;Pisarev R V, Moskvin A S, Kalashnikova A M and Rasing Th 2009 Phys. Rev. B 79 235128;Moskvin A S and Pisarev R V 2010 Low Temp. Phys. 36613 